\title{
Influencia del porcentaje de relleno en la resistencia mecánica en impresión 3D, por medio del método de Modelado por Deposición Fundida (FDM)
}

\author{
Influence of infill parameter on the mechanical resistance in $3 D$ printing, \\ using the Fused Deposition Modeling method \\ Kenny L. Alvarez C. ${ }^{1 *} \quad$ Rodrigo F. Lagos C. ${ }^{1} \quad$ Miguel Aizpun $^{1}$ \\ Recibido 11 de enero de 2016, aceptado 15 de abril de 2016 \\ Received: January 11, 2016 Accepted: April 15, 2016
}

\begin{abstract}
RESUMEN
La impresión 3D es un proceso de manufactura que se basa en la fabricación de prototipos, partes y piezas funcionales. Existen diferentes métodos, en los cuales se utilizan distintos materiales en diversos formatos. Uno de los métodos más utilizados es el Modelado por Deposición Fundida (FDM).

A pesar de las ventajas que posee con respecto a otros procesos de fabricación, la impresión 3D no está libre de dificultades o problemas. Una de las principales problemáticas se presenta al momento de configurar una impresión y tiene relación con la elección de los parámetros de impresión. En ocasiones, la elección se realiza en función de la experiencia de los operadores, pero cuando se requieren propiedades específicas, no se conocen los parámetros a elegir.

En este trabajo, se analizó la influencia del porcentaje de relleno en la resistencia a la tracción de piezas fabricadas en ABS, con una impresora Makerbot Replicator 2X. Para ello, se imprimieron probetas para ensayos de tracción, variando el porcentaje de relleno, manteniendo los demás parámetros constantes. Luego fueron ensayadas y de esta manera se logró obtener la propiedad mecánica de resistencia a la tracción. Además, se analizó el tiempo efectivo de impresión, logrando establecer un rango recomendado de impresión en función de este parámetro combinado con la resistencia a la tracción.

La máxima resistencia a la tracción promedio, se obtuvo con un porcentaje de relleno de $100 \%$ y fue de $34,57[\mathrm{MPa}]$.
\end{abstract}

Palabras clave: Impresión 3D, FDM, ABS, Makerbot Replicator 2X, resistencia a la tracción.

\begin{abstract}
$3 D$ printing is manufacturing process that consists of the fabrication of prototypes, parts and functional pieces. Several methods can be used, with different materials in different formats. One of the most popular techniques is the Fused Deposition Modelling (FDM). This method has several advantages with respect to other manufacturing processes, although it also has some problems and difficulties. One of these problems arises when setting the printing parameters. Generally, the printing parameters are selected according to the user's experience, although it is rather difficult to adapt the parameter settings in order to obtain specific mechanical properties of the printed parts.

This work, analyzes the influence of infill on the tensile strength in parts printed with ABS, using a Makerbot Replicator $2 X 3 D$ printer. Test specimens were printed, modifying the infill, while maintaining the other parameters constant. Afterwards, the test specimens were tested in a traction test obtaining the tensile strength. In addition, the effective impression time was analyzed, in order to obtain a recommended infill ranges of impression time and tensile strength.

Results showed that the maximum tensile strength was 34.57 [MPa] considering a 100\% infill.
\end{abstract}

Keywords: 3D printing, fused deposition modelling, ABS, Makerbot Replicator 2X, mechanical properties.

1 Escuela de Ingeniería Mecánica. Pontificia Universidad Católica de Valparaíso. Av. Los Carrera 01567. Quilpué. Valparaíso, Chile.E-mail: kenny.alvarez@pucv.cl; rodrigo.lagos.cereceda@gmail.com; miguel.aizpun@pucv.cl

* Autor de correspondencia 


\section{INTRODUCCIÓN}

La impresión 3D es un método de fabricación utilizado por diseñadores, científicos, ingenieros, estudiantes, etcétera. Debido a las grandes ventajas que posee con respecto a otras técnicas de manufactura, en la actualidad se utiliza en todas las áreas del conocimiento para el desarrollo de prototipos, partes y piezas. Con el paso de los años, se ha preferido esta técnica de fabricación debido a que se logran obtener piezas terminadas o casi terminadas [1], por lo que se reducen considerablemente los tiempos de fabricación de piezas y/o partes, o simplemente se eliminan los postprocesos que se utilizan para obtener una pieza final. Además de esta cualidad, posee grandes ventajas como la variedad de materiales que se utilizan y la complejidad de las piezas que se pueden fabricar.

A pesar de que la impresión 3D fue descubierta hace poco más de dos décadas, ha sido recientemente cuando ha aumentado de manera exponencial difusión y desarrollo. Esto se debe a los grandes avances que se han observado a nivel de software, hardware y materiales, reduciendo de esta forma los costos de adquisición, que pueden variar desde los USD 700 hasta los USD 10.000 .

Por esta razón, la impresión 3D es objeto de estudio de los economistas a nivel mundial, quienes han evaluado su mercado y han realizado proyecciones en cuanto al crecimiento que tendrá en el futuro. En el 2015 este mercado alcanzó los 3,8 billones de dólares [2], cifra que se verá fuertemente incrementada para el 2021 alcanzando los 10,8 billones de dólares. Por lo tanto, en solo seis años el mercado aumentará en $184 \%$.

Sin embargo, una de las problemáticas, o incertidumbres existentes al momento de fabricar un elemento, es la elección de parámetros de impresión para lograr obtener las propiedades mecánicas deseadas.

El método de Modelado por Deposición Fundida, o más conocido como FDM (Fused Deposition Modeling), es una técnica de tecnología de manufactura por adición de capas que produce piezas y partes con geometrías complejas mediante la extrusión y deposición de un material fundido, normalmente ABS o PLA. El material de impresión que se encuentra inicialmente en forma de filamento se obliga a pasar por una boquilla que se encuentra a altas temperaturas, en donde se funde para luego ser depositado en una plataforma. Por medio de movimientos rectilíneos en un plano, el material va generando la forma de la pieza. Cada pasada corresponde a una línea de material depositado, que se vincula a otra adyacente mediante la energía térmica del material semifundido.

Una vez que la pieza ha sido construida se observa una estructura compuesta por láminas con una estructura anisotrópica [3], por lo que las propiedades mecánicas del producto final no serán las mismas en diferentes direcciones.

Por otra parte, en los procesos de Modelado por Deposición Fundida se pueden controlar las propiedades mecánicas y la calidad superficial, variando los parámetros de impresión, por ejemplo: espesor de capa, dirección de la deposición, número de capas superficiales, distancia entre extrusor y plataforma, etcétera.

A lo largo de los años, investigadores se han encargado de estudiar los parámetros de impresión y evaluar su influencia en las propiedades, calidad superficial, entre otros.

El proceso de fabricación comprende la fusión y solidificación de un material, por lo que durante el proceso se generan esfuerzos residuales. Estos esfuerzos están presentes en el material debido al gradiente de temperatura y a la contracción que ocurre durante el proceso. Se ha analizado la influencia de dos parámetros de impresión en los esfuerzos residuales, la altura de capa y la orientación de construcción, llegando a la conclusión de que los esfuerzos residuales son menores cuando se utiliza una orientación longitudinal $\left(0^{\circ}\right)$ y con un espesor de $0,25 \mathrm{~mm}$ [4].

Por otra parte, se ha observado que los elementos impresos presentan un porcentaje de volumen que no es llenado con material, esto se debe a los cambios bruscos de dirección que posee el proceso, y se encuentra principalmente en las zonas aledañas de la superficie exterior.

Este fenómeno se logra reducir aumentando la temperatura de extrusión. Si esta se aumenta de 
$280{ }^{\circ} \mathrm{C}$ a $290{ }^{\circ} \mathrm{C}$, el volumen de zona no rellena disminuye de $15,86 \%$ a $11,17 \%$, y además si se aumenta la temperatura del entorno desde $70{ }^{\circ} \mathrm{C}$ a $75^{\circ} \mathrm{C}$, el porcentaje de volumen no relleno disminuye aún más, de $11,17 \%$ a 10,34\% (para una temperatura de extrusión constante de $290^{\circ} \mathrm{C}$ ) [5].

La unión de cada filamento se logra por medio de la adhesión de material fundido. Esta adhesión es gradual y depende del tiempo que se le otorgue al proceso y de la temperatura. $\mathrm{Al}$ entrar en contacto el filamento fundido con otro semifundido, se forma un cuello en la interfaz, que crece a medida que pasa el tiempo. Si la temperatura se mantiene elevada por un período más prolongado de lo normal, el cuello crece hasta alcanzar casi el diámetro del filamento [6-7].

Por otra parte, se sabe que la resistencia de esa adhesión es menor que la resistencia del ABS propiamente tal [8-9], por lo que no se espera que las piezas finales posean las mismas propiedades mecánicas que las que posee el ABS.

Avances recientes también han aportado en crear nuevos materiales de impresión, mezclando ABS con partículas de FE y Cu y de esta manera se logra modificar las propiedades mecánicas [10].

En relación a la calidad superficial [11] se han descubierto los principales parámetros que tienen directa relación con la rugosidad. En algunas ocasiones, las piezas requieren de un postprocesado para mejorar la calidad superficial o retirar material sobrante adherido a la superficie de la pieza, lo que conlleva a elevar los costos de fabricación. Esta investigación permitió identificar los parámetros de impresión que otorgan una mayor calidad superficial. Se variaron los parámetros de temperatura de extrusión, altura de capa, orientación de impresión y ancho del filamento fundido. El parámetro más predominante resultó ser la altura de capa y la orientación de la superficie de la pieza. El acabado superficial mejora notablemente con alturas de capas más pequeñas y la menor rugosidad se observó con piezas impresas a $70^{\circ}$ (ver Figura 1). La temperatura de extrusión también posee cierta influencia en la rugosidad, se demostró que a mayor temperatura, la calidad superficial es mejor, debido a que el material fluye de mejor manera, lo que permite una mayor adhesión de las capas.

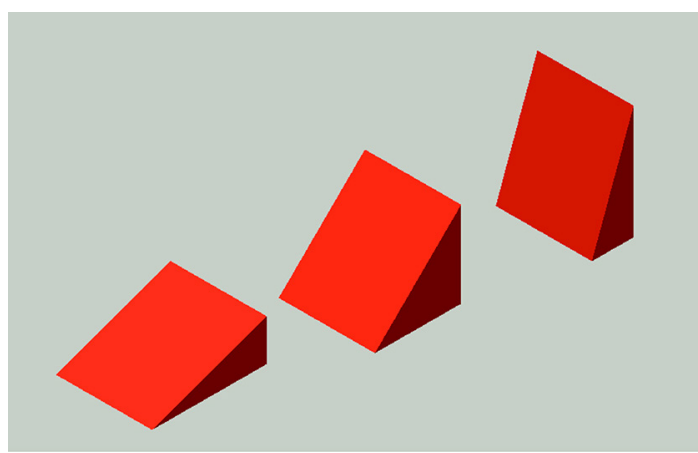

Figura 1. Influencia del ángulo de la superficie (de izquierda a derecha $20^{\circ}, 45^{\circ}$ y $70^{\circ}$ ).

Otro parámetro estudiado es la orientación del filamento durante la construcción de las piezas [12], se estudiaron cuatro diferentes orientaciones $0^{\circ}$, $45^{\circ}, 90^{\circ}$ y $+45^{\circ} / 45^{\circ}$ (ver Figura 2). La resistencia máxima a la tracción resultó con las probetas orientadas en $0^{\circ}$ llegando a una resistencia máxima de 25,72 (MPa). Esto se debe a que la carga aplicada se encuentra en la misma dirección del filamento. La probeta menos resistente fue la que poseía una orientación de $90^{\circ}$.

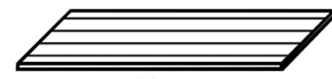

(a)

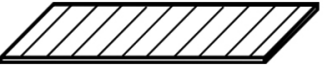

(c)

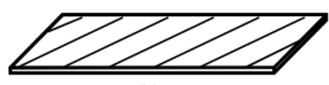

(b)

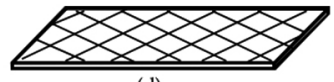

(d)
Figura 2. Orientaciones del filamento en la impresión: a) $0^{\circ}$, b) $45^{\circ}$, c) $90^{\circ}$ y d) $+45^{\circ} \%$ $-45^{\circ}[12]$.

Además de los estudios de parámetros específicos se han realizado análisis de diseño de experimentos factorial [13], para cuantificar el efecto de distintas combinaciones de parámetros de impresión en las propiedades del material, por ejemplo: temperatura, orientación, ancho de filamento, etcétera.

Finalmente [14], se ha logrado caracterizar las propiedades mecánicas de probetas impresas en ABS y PLA, variando los parámetros de altura de capa y orientación de la probeta (manteniendo los otros parámetros constantes con un $100 \%$ de relleno), llegando a descubrir que la orientación de $+45^{\circ} \%$ $-45^{\circ}$ es la que resultó poseer la mayor resistencia 
mecánica en el ensayo de tracción, y la altura de capa de 0,2 fue la que otorgó a las probetas una mayor resistencia. Otro resultado de gran importancia es que las probetas con espesor de capa de 0,4 $\mathrm{mm}$, resultaron tener el mayor módulo elástico, al igual que las probetas orientadas a $0 / 90^{\circ}$.

A pesar de todos los parámetros estudiados por investigadores en el área de la impresión 3D, un parámetro que no ha sido analizado es el porcentaje de relleno.

Al momento de configurar una impresión se debe ingresar el porcentaje de relleno deseado en el elemento a fabricar, es decir, la cantidad de material que los elementos impresos tendrán en su interior. Este parámetro puede tomar valores desde 0 a $100 \%$. Cuando se ingresa $0 \%$ de relleno, la pieza fabricada solo poseerá una superficie externa, sin material en su interior. Si el valor ingresado es mayor a 0 , la máquina depositará material en el interior de las piezas en una forma geométrica específica. La mayoría de las impresoras (incluida la estudiada en este trabajo de investigación) vienen con la forma de construcción hexagonal configurada de fábrica.

El tamaño del hexágono dependerá del porcentaje de relleno que se le ingrese, si el valor del porcentaje de relleno es pequeño, el tamaño de los hexágonos será grande, en cambio cuando se ingresan valores de porcentaje de relleno mayores, los hexágonos serán más pequeños.

\section{DESCRIPCIÓN DEL TRABAJO REALIZADO}

Este trabajo consistió en analizar la influencia del porcentaje de relleno depositado durante la impresión, en las propiedades mecánicas de elementos fabricados por el método de Modelado por Deposición Fundida, en una impresora 3D marca Makerbot, modelo Replicator 2X. Específicamente se analizó la resistencia a la tracción.

Para obtener dichos resultados se modelaron probetas impresas en ABS de acuerdo a las normas ASTM D638-10: "Ensayo de tracción en materiales plásticos" [15], las que fueron ensayadas por medio del ensayo de tracción con el objetivo de obtener la resistencia a la tracción.
Las probetas impresas se fabricaron variando el porcentaje de relleno de 5 en $5 \%$, de 0 a $100 \%$, manteniendo los demás parámetros constantes con los siguientes valores:

- Temperatura plataforma: $125^{\circ} \mathrm{C}$.

- Temperatura extrusión: $250^{\circ} \mathrm{C}$.

- Velocidad de extrusión: $90[\mathrm{~mm} / \mathrm{s}]$.

- Velocidad de avance: 150 [mm/s].

- Numero de capas: 2.

- Altura de capa: 0,2 [mm].

- Diámetro del extrusor: 0,4 [mm].

Se imprimieron cinco especímenes de cada una de las probetas, sumando un total de 105 probetas para ensayo de tracción. La orientación de impresión de las probetas fue en el eje X (ver Figura 3).

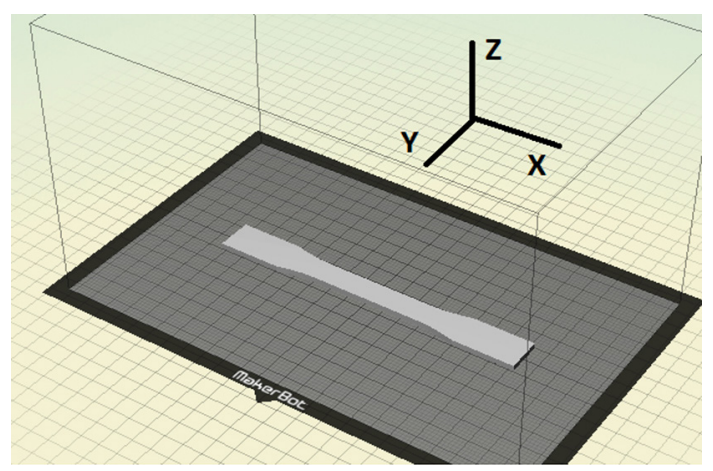

Figura 3. Modelo de probeta para ensayo de tracción.

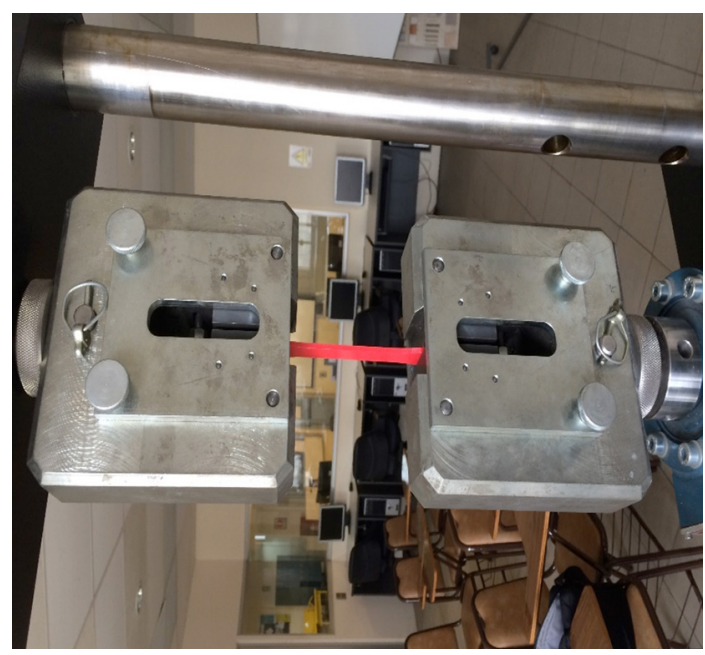

Figura 4. Ensayo de tracción Máquina Universal de ensayos Gunt Hamburg WP 310. 
Los ensayos de tracción se realizaron de acuerdo al procedimiento indicado en la norma ASTM D638-10. La máquina utilizada fue en una Máquina Universal de Ensayos Gunt Hamburg WP 310 de 50 kN. La velocidad de ensayo fue de $2 \mathrm{~mm} / \mathrm{s}$ (ver Figura 4).

\section{RESULTADOS}

A continuación se muestran los resultados obtenidos del ensayo de tracción para las probetas ensayadas:

Tabla 1. Resultados ensayo de tracción.

\begin{tabular}{|c|c|c|c|c|c|}
\hline \multicolumn{6}{|c|}{ Resultados ensayo de tracción } \\
\hline$\stackrel{\Xi}{\Xi}$ & 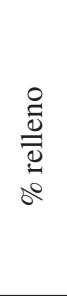 & 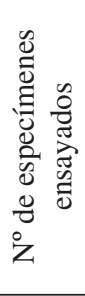 & 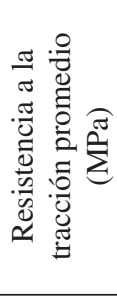 & 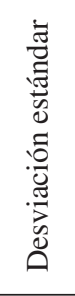 & 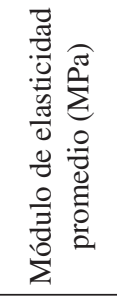 \\
\hline 1 & 0 & 5 & 14,62 & 1,30 & 550,53 \\
\hline 2 & 5 & 5 & 16,54 & 1,51 & 649,69 \\
\hline 3 & 10 & 5 & 16,83 & 2,13 & 600,65 \\
\hline 4 & 15 & 6 & 18,89 & 2,60 & 714,33 \\
\hline 5 & 20 & 6 & 18,61 & 2,55 & 699,44 \\
\hline 6 & 25 & 5 & 18,99 & 2,16 & 747,81 \\
\hline 7 & 30 & 5 & 21,54 & 1,70 & 892,62 \\
\hline 8 & 35 & 5 & 23,22 & 0,55 & 920,74 \\
\hline 9 & 40 & 5 & 21,25 & 0,88 & 796,06 \\
\hline 10 & 45 & 5 & 21,54 & 3,05 & 861,98 \\
\hline 11 & 50 & 5 & 23,51 & 2,24 & 892,44 \\
\hline 12 & 55 & 5 & 23,37 & 2,27 & 872,55 \\
\hline 13 & 60 & 5 & 22,36 & 2,32 & 899,94 \\
\hline 14 & 65 & 5 & 23,80 & 3,63 & 839,88 \\
\hline 15 & 70 & 5 & 23,27 & 2,73 & 877,42 \\
\hline 16 & 75 & 6 & 25,48 & 3,34 & 905,56 \\
\hline 17 & 80 & 5 & 21,30 & 1,43 & 831,90 \\
\hline 18 & 85 & 5 & 21,44 & 0,65 & 856,81 \\
\hline 19 & 90 & 5 & 24,62 & 2,56 & 863,89 \\
\hline 20 & 95 & 5 & 22,93 & 0,44 & 756,56 \\
\hline 21 & 100 & 5 & 34,57 & 0,65 & $1.014,56$ \\
\hline \multicolumn{2}{|c|}{$\begin{array}{c}\text { Total } \\
\text { probetas }\end{array}$} & 108 & & & \\
\hline
\end{tabular}

En la Tabla 1, se pueden observar el número de probetas ensayadas que fueron impresas con el mismo porcentaje de relleno, que en algunos casos fue mayor a cinco. Esto se debe a que la fractura de las probetas no ocurrió en el cuello de menor sección lo que obligó a repetir el ensayo. Se muestra también la resistencia a la tracción promedio, la desviación estándar de cada lote de cinco muestras y, finalmente, el módulo de elasticidad promedio de cada lote.

Con los resultados de las probetas impresas con $100 \%$ de relleno se construyó la curva Resistencia a la Tracción-Deformación Unitaria (ver Gráfico 1).

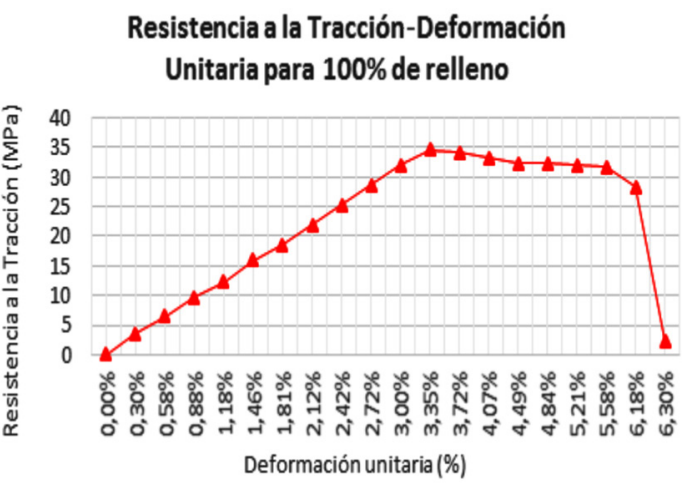

Gráfico 1. Curva Resistencia a la TracciónDeformación Unitaria para 100\% de relleno.

Se observa claramente en esta curva que el material ensayado posee una zona de variación lineal del esfuerzo en función de la deformación. Esta zona corresponde a un comportamiento elástico del material, es decir, si la carga es retirada, el material volverá a su estado original. Luego, la probeta alcanza su resistencia máxima de 34,57 [MPa] en aproximadamente 3\% de deformación. Si la fuerza actuada en la probeta continúa, la resistencia se mantiene prácticamente constante hasta aproximadamente $6 \%$ de deformación, para luego caer bruscamente al ocurrir la fractura del material.

De acuerdo a la literatura [16], la curva de esfuerzodeformación del ABS tiene la misma tendencia que la obtenida en este análisis; sin embargo, la resistencia máxima del ABS no es la misma que la obtenida en los ensayos [8-9].

En el Gráfico 2 se muestra la curva obtenida de Resistencia a la tracción-Porcentaje de relleno. En ella se puede distinguir que a mayor porcentaje de 
relleno, la resistencia a la tracción aumenta, lo que era de esperarse en realidad ya que las probetas que poseen mayor porcentaje de relleno, poseen un área resistente mayor. En algunos casos, al pasar de un porcentaje de relleno menor a uno mayor, la resistencia se mantuvo constante, e incluso disminuyó una pequeña cantidad. Esto se debe principalmente a que el proceso está compuesto por muchas variables que no se pueden controlar con precisión durante el transcurso de este. Por ejemplo, distancia entre el extrusor y la plataforma, alineación de los cabezales, nivelación de la plataforma, temperatura uniforme en la plataforma, etcétera. Cada uno de estos parámetros puede provocar que la fabricación sea distinta entre una probeta y otra, como también que la fabricación sea distinta entre dos o más probetas que sean impresas al mismo tiempo en diferente posición en la plataforma.

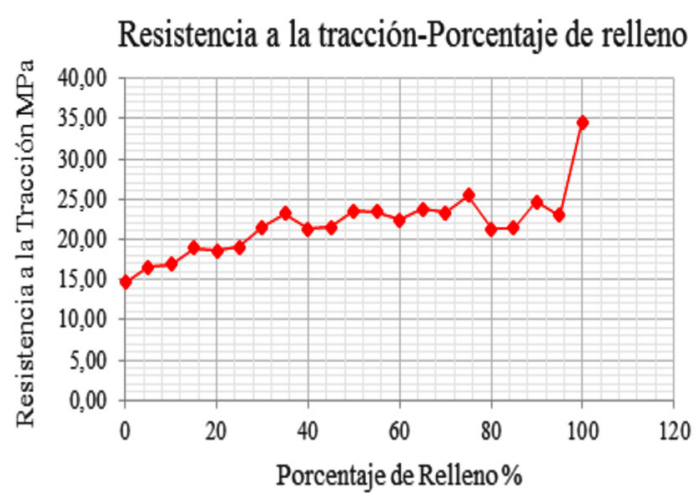

Gráfico 2. Curva Resistencia a la tracción-Porcentaje de relleno.

Otro aspecto importante que se puede observar en la curva anterior, es que la resistencia a la tracción aumenta en pequeñas cantidades mientras aumenta el porcentaje de relleno. Esta tendencia se mantiene prácticamente constante hasta el valor de $95 \%$. Al pasar de 95 a 100\% de relleno, la resistencia aumenta de 22,93 (MPa) a 34,57 (MPa), es decir, aumenta la resistencia a la tracción en aproximadamente $53 \%$.

Además de la resistencia a la tracción, otro parámetro que se estudió fue el tiempo efectivo de impresión, es decir, el tiempo que se tardó en imprimir el especimen desde que se inició el proceso. Esta prueba se realizó imprimiendo solo una probeta, registrando al final del proceso el tiempo total de impresión, para luego restarle el tiempo de calentamiento de los extrusores y plataforma.
Los resultados se pueden observar en el Gráfico 3. Se observa un fenómeno particular en los resultados, ya que el tiempo de impresión en probetas con porcentaje de relleno de 0 a $95 \%$, aumenta linealmente, lo que es esperado ya que al aumentar el porcentaje de relleno, aumenta la cantidad de material a aportar $\mathrm{y}$, por lo tanto, los movimientos que realiza la máquina. En cambio, para una impresión con un $100 \%$ de relleno se observa que el tiempo disminuye considerablemente, incluso este llega a tener el mismo valor que el especimen impreso con $45 \%$ de relleno.

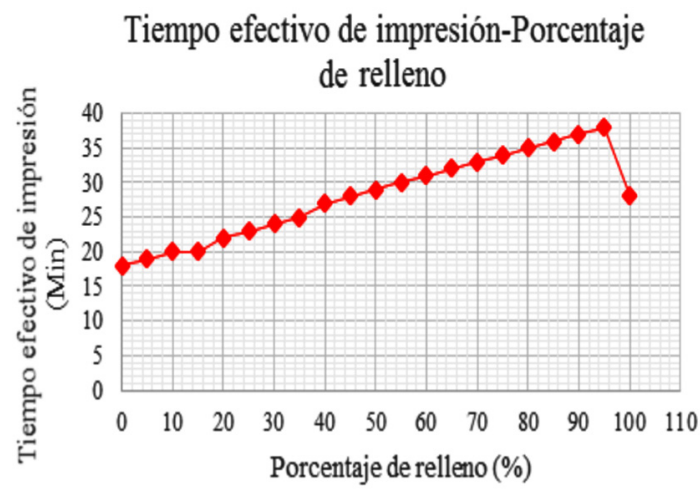

Gráfico 3. Curva tiempo efectivo de impresiónPorcentaje de relleno.

Se investigó para lograr conseguir por qué razón ocurría este fenómeno, para lo cual se observó el proceso de impresión y se logró detectar que la impresión con $100 \%$ de relleno no sigue la misma configuración de movimientos que con menores porcentajes de relleno. Cuando se configura la impresión con $100 \%$ de relleno, la impresora imprime de manera lineal con capas alternadas orientadas en el eje X y otra en el eje Y. Al imprimir de esa forma, la cantidad de movimientos que realiza son menores que cuando imprime de manera hexagonal, y esto se traduce en un menor tiempo de impresión.

\section{CONCLUSIONES}

La impresión 3D es una tecnología que está en constante crecimiento y, por lo tanto, es necesario avanzar en materia de investigación, con el fin de conocer sus capacidades y también sus dificultades, para así poder mejorar el proceso. Este estudio permitió caracterizar la impresora Makerbot Replicator 2X. 
De acuerdo con los resultados obtenidos se logró comprobar que la máxima resistencia en la dirección $\mathrm{X}$ que se puede obtener en elementos fabricados por medio del proceso de FDM, se consigue con $100 \%$ de relleno y es de 34,57 (MPa). Además, se identificó que las propiedades no siempre serán constantes, ya que existen variables que no se pueden controlar en el transcurso de la fabricación.

El otro resultado importante tiene relación con el tiempo de impresión. Generalmente, los usuarios de impresoras seleccionan porcentajes de relleno menores para disminuir los tiempos de impresión, o bien para ahorrar material. Si el motivo es disminuir el tiempo de impresión, no se recomienda imprimir con porcentajes de relleno en un rango de 50 a $95 \%$, ya que el tiempo será mayor que si se imprime con $100 \%$. Además, como se indicó anteriormente, con $100 \%$ se obtendrá la máxima resistencia a la tracción, por lo que no se justifica de ninguna manera imprimir en ese rango de porcentajes de relleno. Queda para futuros estudios analizar si este fenómeno ocurre en todos los modelos y marcas de impresoras, o solo en esta en particular.

\section{AGRADECIMIENTOS}

Este estudio fue posible gracias al financiamiento otorgado por parte del proyecto PMI UCV1301, del Centro de Minería, de la Pontificia Universidad Católica de Valparaíso.

\section{REFERENCIAS}

[1] B. Caulfield, P.E. McHugh and S. Lohfeld. "Dependence of mechanical properties of polyamide components on build parameters in the SLS process". Journal of Materials Processing Technology. Vol. 182, Issues 1-3, pp. 477-488. February, 2007. DOI: 10.1016/j.jmatprotec.2006.09. 007.

[2] Credit Suisse. "3D printing market will be much bigger than what industry consultants estimate". September, 2013. Fecha de consulta: 10 agosto de 2015. URL: http://www.3ders. org/articles/ 20130918-credit-suisse-3dprinting-market-will-be-much-bigger-thanwhat-industry-consultants-estimate.html

[3] O.S. Es-Said, J. Foyos, R. Noorani, M. Mendelson, R. Marloth and B.A. Pregger. "Effect of Layer Orientation an Mechanical
Properties of Rapid Prototyped Samples". Materials and Manufacturing Processes. Vol. 15, Issue 1, pp. 107-122. 2000. DOI: 10.1080/10426910008912976.

[4] A. Kantaros and D. Karalekas. "Fiber Bragg grating based investigation of residual strains in ABS parts fabricated by fused deposition modeling process". Materials and Design. Vol. 50, pp. 44-50. Sep. 2013. ISSN: 0264-1275. DOI: 10.1016/j. matdes.2013.02.067.

[5] I.Gajdoš and J. Slota. "Influence of printing conditions on structure in FDM prototypes". Technical Gazette. Vol. 20, Issue 2, pp. 231236. 2013. DOI: 658.512.2:004.896.

[6] C. Bellehumeur, L. Li, Q. Sun and P. Gu. "Modeling of Bond Formation between Polymer Filaments in the Fused Deposition Modeling Process". Journal of Manufacturing Processes. Vol. 6, Issue 2, pp. 170-178. 2004. ISSN: 1526-6125. DOI: 10.1016/ S1526-6125(04)70071-7.

[7] L. Li, Q. Sun, C. Bellehumeur and P. Gu. "Investigation of bond formation in FDM process". Solid Freegorm Fabrication Symp., Austin, TX, USA. Issue 403, pp. 400-407. August, 2001. ISSN: 10473025.

[8] L. Li, Q. Sun, C. Bellehumeur and P. Gu. "Composite Modeling and Analysis of FDM Prototypes for Design and Fabrication of Functionally Graded Parts". Solid Freegorm Fabrication Symp., pp. 187-194, Austin, TX, USA, 2001.

[9] L. Rodriguez, J. Thomas and J. Renaud. "Mechanical Behavior of Acrylonitrile Butadiene Styrene (ABS) Fused Deposition Materials". Rapid Prototyping Journal. Vol. 7 $\mathrm{N}^{\circ} 3$, pp. 148-158. 1995. ISSN: 1355-2546. DOI: $10.1108 / 13552540310489604$.

[10] M. Nikzad, S.H. Masood and I. Sbarski. "Thermo-mechanical properties of a highly filled polymeric composites for Fused Deposition Modeling". Materials and Design. Vol. 32, Issue 6, pp. 3448-3456. June, 2011. ISSN: 02641275. DOI: 10.1016/j. matdes.2011.01.056.

[11] B. Vasudevarao, D. Prakash Natarajan and M. Henderson. "Sensitivitiy of RP Surface Finish to Process Parameter Variation". Solid Freeform Fabrication Proceedings, pp. 251-258. 2000. 
[12] C. Ziemian and M. Sharma. "Anisotropic mechanical properties of ABS parts fabricated by fused deposition modelling". Mechanical Engineering, pp. 159-180. 2012. DOI: 10.5772/34233.

[13] J. Weinmann, H. Ip, D. Prigozhin, E. Escobar, M. Mendelson and R. Noorani. "Applicaton of Design of Experiments (Doe) on the Processing of Rapid Prototyped Samples". The Solid Freeform Symposium, Proceedings, pp. 340-347, Austin, Texas, USA, 2003.
[14] B.M. Tymrak, M. Kreiger and J.M. Pearce. "Mechanical properties of components fabricated with open-source 3-D printers under realistic environmental conditions". Materials and Design. Vol. 58, pp. 242-246. June, 2014. ISSN: 18734197. DOI: 10.1016/j. matdes.2014.02.038.

[15] ASTM D638-10. "Standard test Method for Tensile Properties of Plastics".

[16] Erik Lokensgard. "Industrial Plastics: Theory and Aplications". 5th Edition. 560 páginas. 2010. ISBN: $1435486161,9781435486164$. 